\title{
Stable Filtering Procedures for Nodal Discontinuous Galerkin Methods
}

\author{
Jan Nordström ${ }^{1,2}$ (D) Andrew R. Winters ${ }^{1}$ \\ Received: 22 July 2020 / Revised: 18 November 2020 / Accepted: 13 February 2021 / \\ Published online: 27 February 2021 \\ (c) The Author(s) 2021
}

\begin{abstract}
We prove that the most common filtering procedure for nodal discontinuous Galerkin (DG) methods is stable. The proof exploits that the DG approximation is constructed from polynomial basis functions and that integrals are approximated with high-order accurate Legendre-Gauss-Lobatto quadrature. The theoretical discussion re-contextualizes stable filtering results for finite difference methods into the DG setting. Numerical tests verify and validate the underlying theoretical results.
\end{abstract}

Keywords Discontinuous Galerkin · Filtering · Stability · Transmission problem

\section{Introduction}

High frequency errors are always present in numerical simulations due to inaccuracies of the spatial derivatives at high-wave numbers. A common technique to combat such errors is to use a filter operator that removes high wave number oscillations, e.g. [7, Sect. 5.3]. The filtering procedure is separate from the scheme itself, and is often done in an ad hoc fashion. Filtering is applied "as little as possible, but as much as needed".

Recently, Lundquist and Nordström [11] removed the ad hoc nature of filtering in finite difference (FD) methods. They derive a contractivity condition on the explicit filter matrix by re-framing the filtering procedure as a transmission problem [13]. The work in [11] develops a necessary condition for the filter matrix and how it must be related to the particular discrete quadrature rule.

The goal of the present work is to remove the ad hoc nature of the nodal DG filtering procedure and prove that the commonly used explicit filter technique from the spectral community $[5,6]$ is stable. Just as in the case of FD methods, the stability of the filtering for

Jan Nordström

jan.nordstrom@liu.se

Andrew R. Winters

andrew.ross.winters@liu.se

1 Department of Mathematics; Computational Mathematics, Linköping University, SE-581 83 Linköping, Sweden

2 Department of Mathematics and Applied Mathematics, University of Johannesburg, P.O. Box 524, Auckland Park 2006, Johannesburg, South Africa 
nodal DG relies upon the existence of a high-accuracy auxiliary filter matrix as well as a semi-discrete bound on the solution.

The remainder of the paper is organized as follows: Sect. 2 gives an overview of the nodal DG method and the commonly used filtering procedure. Section 3 generalizes the theoretical stability results from [11] into the DG context. Numerical results that verify the theoretical findings are found in Sect. 4. Concluding remarks are given in Sect. 5.

\section{Overview of Nodal DG Approximation}

DG methods are principally designed to approximate solutions of hyperbolic conservation laws, see [7, Chap. 2] or [9, Sect. 4.8] for details. Here, we consider such a conservation law in one spatial dimension

$$
\frac{\partial u}{\partial t}+\frac{\partial f}{\partial x}=0, \quad x \in\left[x_{L}, x_{R}\right]
$$

where $u \equiv u(x, t)$ is the solution and $f \equiv f(u)$ is the flux function. The conservation law is then equipped with an initial condition $u(x, 0) \equiv u_{\text {ini }}(x)$ and boundary condition(s).

To arrive at the nodal DG approximation we first transform the domain $\left[x_{L}, x_{R}\right]$ to the reference interval $[-1,1]$. To do so, we apply an affine mapping $\xi(x) \in[-1,1][9$, Eq. 8.10] and rewrite the conservation law in the computational coordinate $\xi$. The nodal DG approximation is built from the weak form of the mapped equation. The solution and fluxes are approximated by Lagrange polynomials of degree $N$ and the inner products in the variational form are approximated with high-order Legendre-Gauss-Lobatto (LGL) quadrature. Full details are given in [9, Sect. 4.7].

The resulting strong form, nodal DG approximation is then

$$
\frac{\Delta x}{2} \underline{U}+\mathcal{D} \underline{F}+\mathcal{M}^{-1} \mathcal{B}\left(\underline{F}^{*}-\underline{F}\right)=0
$$

where $\underline{U}=\left[U_{0}, U_{1}, \ldots, U_{N}\right]^{T}$ is the vector form for the degrees of freedom and $\underline{F}$ is the discrete flux. The matrices in (2.1) are the discrete derivative matrix [9, Sect. 3.5.2] $\mathcal{D}_{i j}=\ell_{j}^{\prime}\left(\xi_{i}\right)$ where $\left\{\ell_{j}(\xi)\right\}_{j=0}^{N}$ are the nodal Lagrange basis polynomials and $\left\{\xi_{i}\right\}_{i=0}^{N}$ are the LGL quadrature nodes, the mass matrix $\mathcal{M}=\operatorname{diag}\left(\omega_{0}, \ldots, \omega_{N}\right)$ containing the LGL quadrature weights and the boundary matrix $\mathcal{B}=\operatorname{diag}(-1,0, \ldots, 0,1)$. There exist several numerical flux functions depending on the particular mathematical flux function $f(u)$, see Toro [16, Chap. 2] for details. The numerical flux used in this work is the Lax-Friedrichs flux, $F^{*}=\left(F^{R}+F^{L}-\lambda_{\max }\left(U^{R}-U^{L}\right)\right) / 2$ where $\lambda_{\max }$ is the largest wave speed, see [4, Sect. 4.2] or [16, Sect. 5.3.4] for details.

Two features to note for this flavour of nodal DG approximation are: Firstly, the diagonal mass matrix $\mathcal{M}$ denotes a quadrature rule that is exact for polynomials up to degree $2 N-1$. So, there is equality between the continuous and discrete integral

$$
\langle V, W\rangle_{N}=\underline{V^{T}} \mathcal{M} \underline{W}=\sum_{i=0}^{N} V_{i} \mathcal{M}_{i i} W_{i}=\int_{-1}^{1} v w \mathrm{~d} \xi=\langle v, w\rangle
$$

provided the product of the functions $v$ and $w$ is a polynomial of degree $\leq 2 N-1$. From (2.2), the continuous and discrete $L_{2}$ norms are $\langle v, v\rangle=\|v\|^{2}$ and $\langle V, V\rangle_{N}=\|V\|_{N}^{2}$. Secondly, the mass and derivative matrices of the LGL collocation DG scheme (2.1) form a summation-by-parts (SBP) operator pair [14] for any nodal polynomial order $N[4$, Sect. 
2.1], i.e. $\mathcal{M D}+(\mathcal{M D})^{T}=\mathcal{B}$. From the SBP property, stable versions of the nodal DG method can be constructed $[3,4]$.

\subsection{Construction of Filtering for Nodal DG}

The general idea of filtering in DG exploits that the polynomial representation of the function $U$ is unique, and hence can be written in terms of other basis polynomial functions. At present, we select the modal (normalized) hierarchical Legendre basis polynomials $\left\{L_{j}(\xi)\right\}_{j=0}^{N}$ to express the function $U$ in a modal polynomial basis. It is straightforward to compute the Vandermonde matrix $\mathcal{V}$ associated with the LGL nodal interpolation nodes $\mathcal{V}_{i j}=L_{j}\left(\xi_{i}\right)$, with $i, j=0, \ldots, N$, which allows us to transform the nodal degrees of freedom, $\left\{U_{i}\right\}_{i=0}^{N}$, to modal degrees of freedom, $\left\{\widetilde{U}_{j}\right\}_{j=0}^{N}$ and vice versa, i.e., $\underline{U}=\mathcal{V} \underline{\widetilde{U}}$ and $\underline{\widetilde{U}}=\mathcal{V}^{-1} \underline{U}$. Filtering in modal space is performed with a diagonal cutoff matrix $\mathcal{C}_{i j}=\delta_{i j} \sigma_{i}$ [5, Eq. 23], such that a typical filter matrix becomes $\mathcal{F}=\mathcal{V} \mathcal{C} \mathcal{V}^{-1}$.

Vandeven [17, Theorem 3] states that a filter function $\sigma(\eta): \mathbb{R}^{+} \mapsto[0,1]$ should be

- $\sigma$ is $(s-1)$-times continuously differentiable in $\mathbb{R}$.

- $\sigma(0)=1$ and $\sigma^{(k)}(0)=0$ for $k=1, \ldots, s-1$.

- $\sigma(\eta)=0$ if $\eta \geq 1$.

In the nodal DG community a typical choice to define the coefficients $\sigma_{i}$ is [7]

$$
\sigma_{i}= \begin{cases}1 & \text { if } 0 \leq i \leq N_{c}-1 \\ \exp \left(-\alpha\left(\frac{i+1-N_{c}}{N+1-N_{c}}\right)^{s}\right) & \text { if } N_{c} \leq i \leq N\end{cases}
$$

where $\alpha, s$ and $N_{c}$ are the filter parameters. The value $N_{c}$ indicates the number of the unaffected modes, $\alpha$ is chosen such that $\exp (-\alpha)$ is machine epsilon and $s$ is an even number determining the strength of the filter. For any choice of the filter parameters the filter coefficients are constructed such that $0 \leq \sigma_{i} \leq 1$. This exponential filter does not strictly adhere to Vandeven's definition of the filter function [17, Theorem 3], but it does so in practice by choosing $\alpha$ such that $\sigma_{N}$ is below machine accuracy [1, Sect. 2.1.4]. This filter matrix retains the high-order accuracy of the nodal DG approximation for smooth functions [6, Chap. 5], [1, Sect. 7.6.3]. The accuracy of the filter $\mathcal{F}$ lies entirely in the filter function $\sigma$ used to create the diagonal entries in the matrix $\mathcal{C}$, as shown by Vandeven [17, Theorem 3].

Remark 1 Filtering has often been used as a stabilization technique for numerical methods such as in FD [8] as well as DG [5, Sect. 2.3] methods. We strongly advise against such use. Instead, one should first construct construct a (provably) stable numerical scheme. After this, the solution quality can be addressed and cleaned-up, possibly using filtering.

\section{Stability}

Filtering is separate from the spatial discretization and changes the approximate solution during the time integration procedure. To discuss the filtering and its effect on stability we follow [11] into the nodal DG context. With homogeneous boundary conditions, semidiscrete stability ensures that the discrete norm of the approximate solution is bounded by the discrete norm of the initial conditions, see [12, Sect. 4] for complete details. For the nodal DG approximation such a stability statement takes the form $\|U(t)\|_{N} \leq\left\|U_{\text {ini }} \mid\right\|_{N}$, where $U_{\text {ini }}$ is the initial condition evaluated at the LGL nodes. 
Pursuant to the work [11], we view the application of a filter matrix to a discrete solution at some intermediate time $t_{1}$ as a transmission problem [13, Sect. 3]:

$$
\begin{aligned}
& \underline{U}_{t}+\mathbb{D}(\underline{U})=0, \quad 0 \leq t \leq t_{1}, \quad \underline{U}(0)=\underline{U}_{\mathrm{ini}}, \\
& \underline{V}_{t}+\mathbb{D}(\underline{V})=0, \quad t \geq t_{1}, \quad \underline{V}\left(t_{1}\right)=\mathcal{F} \underline{U}\left(t_{1}\right)
\end{aligned}
$$

where the operator $\mathbb{D}$ contains the derivative matrix $\mathcal{D}$ as well as the boundary conditions. The filtering stated in the final term of (3.1) is performed in an explicit fashion. For stability it must hold that the filter is contractive, i.e. $\left\|V\left(t_{1}\right)\right\|_{N} \leq\left\|U\left(t_{1}\right)\right\|_{N}$. In turn, this contractive property guarantees that the filter procedure is stable because $\left\|V\left(t_{1}\right)\right\|_{N} \leq\left\|U\left(t_{1}\right)\right\|_{N} \leq$ $\left\|U_{\text {ini }}\right\|_{N}$.

The contractivity property implies that the following contractivity condition [11, Eq. 7] on the explicit filter matrix $\mathcal{F}$ must hold

$$
\mathcal{F}^{T} \mathcal{M} \mathcal{F}-\mathcal{M} \leq 0 .
$$

The contractivity condition (3.2) expresses a precise interplay between the filter matrix and the mass matrix. A necessary condition for the explicit filter matrix to satisfy (3.2) is that an auxiliary filter matrix $\widetilde{\mathcal{F}}_{\text {aux }}=\mathcal{M}^{-1} \mathcal{F}^{T} \mathcal{M}$, exists and possesses the same accuracy as $\mathcal{F}$, otherwise (3.2) is provably indefinite [11, Sect. 4.1].

We will show that the auxiliary filter matrix is identical to the original filter matrix for the LGL nodal DG approximation. Furthermore, the filter matrix $\mathcal{F}$ indeed satisfies the contractivity condition (3.2). Both results require the following Lemma.

Lemma 1 The matrix product $\mathcal{V}^{T} \mathcal{M V}$ is the LGL quadrature rule applied to the (normalized) Legendre polynomial functions $\left\{L_{j}(\xi)\right\}_{j=0}^{N}$ and results in a diagonal matrix

$$
\mathcal{V}^{T} \mathcal{M} \mathcal{V}=\operatorname{diag}(1,1, \ldots, 1,2+1 / N):=\mathcal{K}
$$

Proof The entries of this matrix product in terms of discrete inner products is

$$
\mathcal{V}^{T} \mathcal{M} \mathcal{V}=\left[\begin{array}{cccc}
\left\|L_{0}\right\|_{N}^{2} & \left\langle L_{0}, L_{1}\right\rangle_{N} & \cdots & \left\langle L_{0}, L_{N}\right\rangle_{N} \\
\left\langle L_{1}, L_{0}\right\rangle_{N} & \left\|L_{1}\right\|_{N}^{2} & \cdots & \left\langle L_{1}, L_{N}\right\rangle_{N} \\
\vdots & \vdots & \ddots & \vdots \\
\left\langle L_{N}, L_{0}\right\rangle_{N} & \left\langle L_{N}, L_{1}\right\rangle_{N} & \cdots & \left\|L_{N}\right\|_{N}^{2}
\end{array}\right] .
$$

From the accuracy of the LGL quadrature and the fact that $\left\{L_{j}(\xi)\right\}_{j=0}^{N}$ are polynomials, we have equality between the discrete and continuous inner products

$$
\left\langle L_{j}, L_{k}\right\rangle_{N}=\left\langle L_{j}, L_{k}\right\rangle=\left\|L_{j}\right\|^{2} \delta_{j k}=\delta_{j k},
$$

provided $j+k \leq 2 N-1$. The result above utilizes that the Legendre basis is orthonormal. The quadratures are therefore exact for all inner products except the one related to $L_{N}$ with itself since $2 N>2 N-1$. Thus, $\mathcal{V}^{T} \mathcal{M} \mathcal{V}=\operatorname{diag}\left(1,1, \ldots, 1,\left\|L_{N}\right\|_{N}^{2}\right)$. The discrete and continuous norms are equivalent in that when $\phi$ is a polynomial of degree $N$, the discrete and continuous $L^{2}$ norms are related by $\|\phi\|_{N}=\sqrt{2+1 / N}\|\phi\|$ for the LGL quadrature [2, Lemma 3.2]. From this, $\mathcal{V}^{T} \mathcal{M} \mathcal{V}$ equals the diagonal matrix $\mathcal{K}$.

We can now prove

Proposition 1 The auxiliary filter is identical to the DG filter matrix, i.e. $\widetilde{\mathcal{F}}_{\text {aux }}=\mathcal{F}$. 
Proof We examine the difference between the two filter matrices

$$
\widetilde{\mathcal{F}}_{\text {aux }}-\mathcal{F}=\mathcal{M}^{-1} \mathcal{F}^{T} \mathcal{M}-\mathcal{F}=\mathcal{M}^{-1} \mathcal{V}^{-T} \mathcal{C} \mathcal{V}^{T} \mathcal{M}-\mathcal{V} \mathcal{C} \mathcal{V}^{-1}
$$

Next, we factor out the matrix $\mathcal{V}$ on the left and $\mathcal{V}^{-1}$ on the right to have

$$
\widetilde{\mathcal{F}}_{\text {aux }}-\mathcal{F}=\mathcal{V}\left[\left(\mathcal{V}^{-1} \mathcal{M}^{-1} \mathcal{V}^{-T}\right) \mathcal{C}\left(\mathcal{V}^{T} \mathcal{M V}\right)-\mathcal{C}\right] \mathcal{V}^{-1}=\mathcal{V}\left[\left(\mathcal{V}^{T} \mathcal{M V}\right)^{-1} \mathcal{C}\left(\mathcal{V}^{T} \mathcal{M V}\right)-\mathcal{C}\right] \mathcal{V}^{-1}
$$

Applying the result from Lemma 1 gives

$$
\widetilde{\mathcal{F}}_{\text {aux }}-\mathcal{F}=\mathcal{V}\left[\mathcal{K}^{-1} \mathcal{C K}-\mathcal{C}\right] \mathcal{V}^{-1}=0,
$$

where we use that the matrices $\mathcal{C}$ and $\mathcal{K}$ are diagonal to obtain the desired result.

The following result is then self-evident.

Corollary 1 The auxiliary filter $\widetilde{\mathcal{F}}_{\text {aux }}$ exists and is as accurate as $\mathcal{F}$.

Further, the result from Lemma 1 allows us to prove

Proposition 2 The nodal DG filter matrix $\mathcal{F}$ is contractive in the sense of (3.2).

Proof We substitute the form of the filter matrix into the condition (3.2) to obtain

$$
\mathcal{F}^{T} \mathcal{M} \mathcal{F}-\mathcal{M}=\left(\mathcal{V} \mathcal{C} \mathcal{V}^{-1}\right)^{T} \mathcal{M}\left(\mathcal{V} \mathcal{C} \mathcal{V}^{-1}\right)-\mathcal{M}=\left(\mathcal{V}^{-1}\right)^{T} \mathcal{C}\left(\mathcal{V}^{T} \mathcal{M} \mathcal{V}\right) \mathcal{C} \mathcal{V}^{-1}-\mathcal{M}
$$

The middle term, $\mathcal{V}^{T} \mathcal{M} \mathcal{V}$, grouped above is precisely that from Lemma 1, which gives

$$
\mathcal{F}^{T} \mathcal{M} \mathcal{F}-\mathcal{M}=\left(\mathcal{V}^{-1}\right)^{T} \mathcal{C} \mathcal{K} \mathcal{C} \mathcal{V}^{-1}-\mathcal{M}=\left(\mathcal{V}^{-1}\right)^{T} \mathcal{C}^{2} \mathcal{K} \mathcal{V}^{-1}-\mathcal{M}
$$

where we use that the matrices $\mathcal{C}$ and $\mathcal{K}$ are diagonal. From (3.3), the contractivity condition then becomes

$$
\mathcal{F}^{T} \mathcal{M} \mathcal{F}-\mathcal{M}=\left(\mathcal{V}^{-1}\right)^{T} \mathcal{C}^{2} \mathcal{K} \mathcal{V}^{-1}-\mathcal{M}=\left(\mathcal{V}^{-1}\right)^{T}\left(\mathcal{C}^{2}-\mathcal{I}\right) \mathcal{K} \mathcal{V}^{-1}
$$

Recall that the modal filter matrix $\mathcal{C}$ is diagonal with entries $\left\{\sigma_{i}\right\}_{i=0}^{N}$ and, by construction, the term $\sigma_{N}=e^{-\alpha} \approx 0$. Therefore,

$$
\mathcal{C}^{2}-\mathcal{I}=\operatorname{diag}\left(\sigma_{0}^{2}-1, \sigma_{1}^{2}-1, \ldots, \sigma_{N-1}^{2}-1, e^{-\alpha}-1\right) \leq 0
$$

because $0 \leq \sigma_{i} \leq 1$ for $i=0, \ldots, N$. Thus, (3.2) holds.

Remark 2 The proof above holds provided the filter function is chosen such that $\sigma(\eta) \in[0,1]$. Thus, other proposed filter functions like those given in Vandeven [17, Remark 5] also produce a provably stable filter matrix.

The discussions above are for a single spectral element. However, the contractive filter matrix $\mathcal{F}$ and its stability carries through to a multi-element DG approximation. At a given time and applied locally within each element, the filtering in a DG formulation is stable. This is because the underlying spatial approximation is constructed to be stable, e.g. [4,10]. Therefore, the overall discretization in a given time step is, for example: (1) Filter the current solution at time $t_{n}$ in each element using the above described filter; (2) Apply the stable DG coupling of the elements by numerical flux functions (3) Evolve the solution in all elements one time step to $t_{n+1}$; (4) Repeat the procedure from (1). Such an algorithm retains a semidiscrete stability estimate.

Remark 3 A crucial component in order for the filtering procedure to be stable in a semidiscrete sense is that the underlying spatial approximation is stable. 

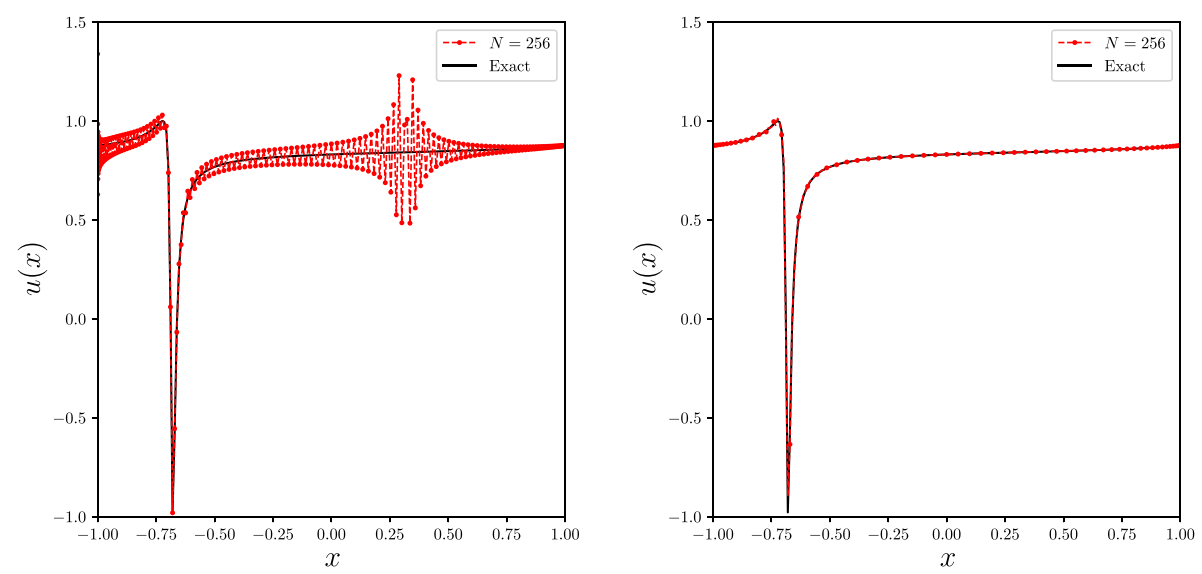

Fig. 1 Unfiltered (left) and filtered (right) nodal DG solution for the variable wave speed problem at $T=4$ with polynomial order $N=256$

\section{Numerical Results}

We consider two test problems that develop a non-smooth solution over time and select $\alpha=36, N_{c}=4$ and $s=16$ for the filter parameters in (2.3). To integrate the semidiscrete DG method (2.1) in time, we use a third-order, low storage Runge-Kutta scheme of Williamson [18, Table 1].

As a first example we consider a variable coefficient linear advection problem, $u_{t}+$ $a(x) u_{x}=0$, with a bounded solution that develops steep gradients [6, Sect. 3.2]. The variable wave speed is $a(x)=\sin (\pi x-1) / \pi$ on the domain $\Omega=[-1,1]$. The wave speed $a(x)$ is positive at the boundaries of the domain. Therefore, no boundary condition is set at the right and at the left the boundary condition is enforced weakly through the numerical flux function and the prescription of an external solution state using the exact solution. We take the initial condition $u_{\text {ini }}(x)=\sin (\pi x)$ with the corresponding analytical solution $u(x, t)=$ $\sin \left(2 \tan ^{-1}\left(e^{-t} \tan ((\pi x-1) / 2)\right)+1\right)$ [6, Sect. 3.2]. In Fig. 1 we compare the solutions at time $T=4$ using polynomial order $N=256$ and $\Delta t=1 / 2000$. The unfiltered DG scheme contains spurious oscillations whereas the filtered one does not. The filter matrix was applied to the solution after every time step in the simulation.

The second example illustrates the importance of the stability of the underlying spatial discretization. We consider Burgers' equation and two forms of the nodal DG spatial discretization. One discretizes the conservative form of the PDE where the nonlinear Burgers' flux is $f(u)=u^{2} / 2$ while the other writes the spatial derivative of the flux in a split formulation $f_{x}(u)=\left(\left(u^{2}\right)_{x}+u u_{x}\right) / 3$. On the continuous level these two forms are equivalent; however, on the discrete level they exhibit different behavior. Most notably, the solution energy $u^{2} / 2$ is bounded for the nodal skew-symmetric DG discretization whereas no such bound exists for the discrete conservative form [4, Sect. 4]. If the underlying numerical scheme is provably stable, and the filtering is contractive, it will remove energy from the solution in a stable controlled way. However, if the underlying scheme is unstable the filtering still removes energy and the approximation might seem stable.

To illustrate this we consider the domain $\Omega=[0,2]$ with periodic boundary conditions and the initial condition $u_{\text {ini }}(x)=\frac{1}{5}[1+\cos (\pi x)]$. We run the simulation with polynomial order $N=128$ up to $T=2.25$ and filter the solution at 16 equally spaced times. We run four 


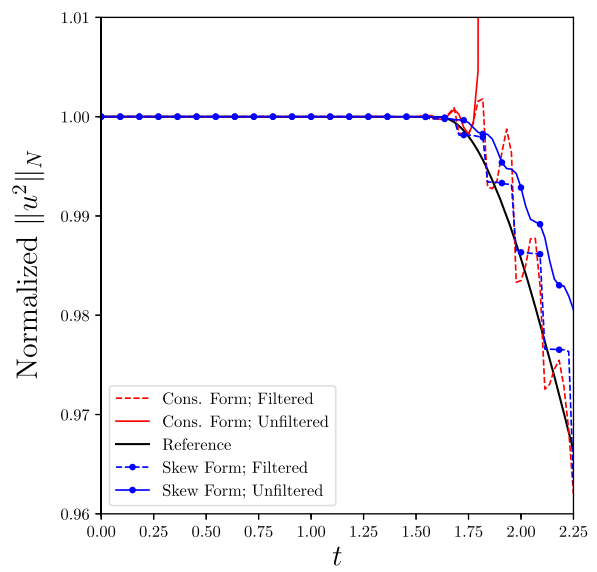

(a)

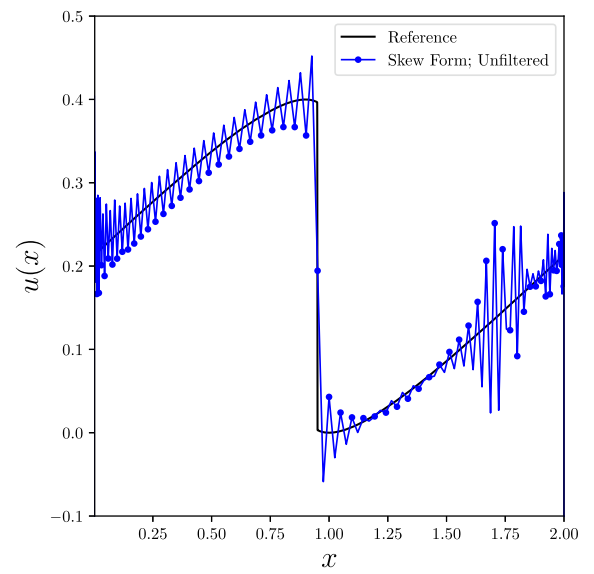

(c)

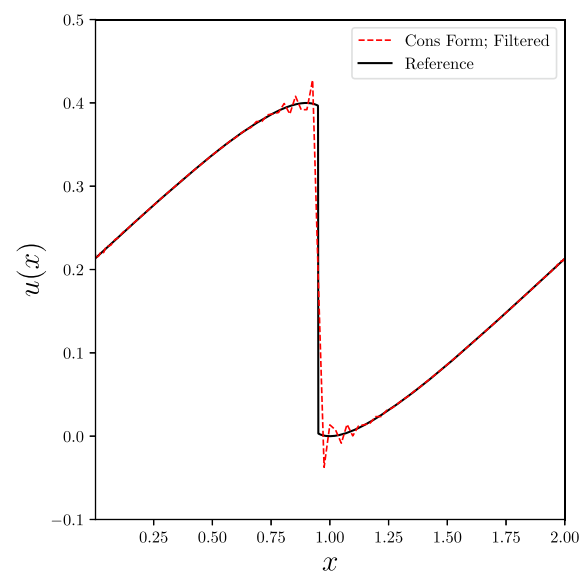

(b)

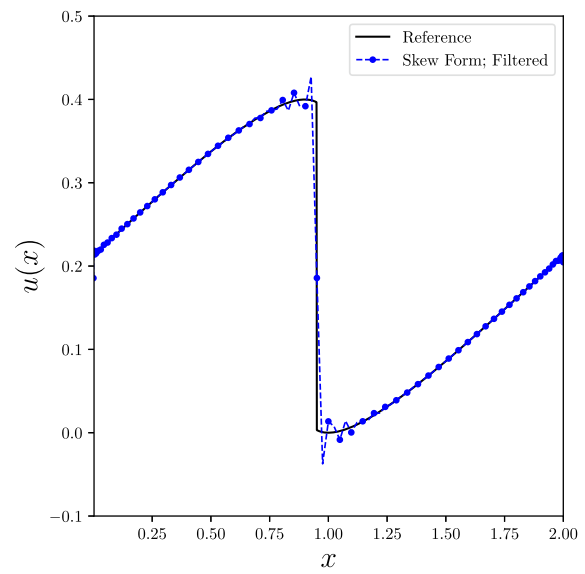

(d)

Fig. 2 a Solution energy evolution of four nodal DG variants for Burgers' equation. The approximate solution $(N=128)$ and a reference finite volume solution are given at $T=2.25$ for the $\mathbf{b}$ filtered conservative form, c unfiltered skew-symmetric form and $\mathbf{d}$ filtered skew-symmetric form

variants of the nodal DG scheme: Conservative formulation; unfiltered and filtered as well as skew-symmetric formulation; unfiltered and filtered. In Fig. 2a we present the evolution of the solution energy, normalized with its initial value. Figure $2 b-d$ present the approximate solution at the final time produced by the filtered conservative, unfiltered skew-symmetric and filtered skew-symmetric DG schemes, respectively. The reference solution is created with a standard finite volume scheme on 10,000 grid cells.

The simulation is well resolved and the four variants are nearly indistinguishable for most of the simulation time. However, as the gradients steepen we see that the conservative formulation, for which no energy stability exists, behave erratically. The unfiltered conservative simulation crashes at $T \approx 1.8$. The filtered conservative simulation runs as the filtering keeps the solution energy "under control." In Fig. 2a we observe growth in the solution energy between the filter applications because the underlying spatial discretization is unstable. The solution energy of unfiltered and filtered skew-symmetric simulations both remain bounded 
because the underlying spatial discretization possesses an energy bound. Comparing Fig. 2b, $\mathrm{d}$, we see at the final time the filtered conservative and skew-symmetric DG schemes produce nearly identical results. As previously noted, the unfiltered conservative DG scheme crashes while the skew-symmetric DG scheme runs. The solution energy $u^{2} / 2$ for Burgers' equation is a conserved quantity for smooth solution and should only dissipate in the presence of discontinuous solutions [4, Sect. 4]. The skew-symmetric DG approximation is constructed to conserve the solution energy $u^{2} / 2$. So, in the absence of dissipation spurious oscillations develop near the discontinuity and propagate throughout the domain as energy is redistributed at the smallest resolvable scale [15, Sect. 6]. Thus, as shown in Fig. 2c, we see that the unfiltered skew-symmetric scheme is stable but the solution quality is extremely poor due to unphysical ringing.

\section{Closing Remarks}

We proved that the commonly used nodal DG filter matrix $\mathcal{F}$ satisfied a contractivity condition. This result implied that the explicit filtering procedure for nodal DG methods is stable. Numerical results verified that the filtering was efficient and stable if the underlying spatial discretization of the method had a semi-discrete bound.

\section{Compliance with Ethical Standards}

Conflict of interest The authors have no relevant financial or non-financial interests to disclose.

Funding Open access funding provided by Linköping University.

Availability of data and material All data generated or analysed during this study are included in this published article.

Code availability The code used to generate the results in this work is available upon request with Andrew Winters (andrew.ross.winters@liu.se).

Open Access This article is licensed under a Creative Commons Attribution 4.0 International License, which permits use, sharing, adaptation, distribution and reproduction in any medium or format, as long as you give appropriate credit to the original author(s) and the source, provide a link to the Creative Commons licence, and indicate if changes were made. The images or other third party material in this article are included in the article's Creative Commons licence, unless indicated otherwise in a credit line to the material. If material is not included in the article's Creative Commons licence and your intended use is not permitted by statutory regulation or exceeds the permitted use, you will need to obtain permission directly from the copyright holder. To view a copy of this licence, visit http://creativecommons.org/licenses/by/4.0/.

\section{References}

1. Canuto, C., Hussaini, M., Quarteroni, A., Zang, T.: Spectral Methods: Fundamentals in Single Domains. Springer, Berlin (2006)

2. Canuto, C., Quarteroni, A.: Approximation results for orthogonal polynomials in Sobolev spaces. Math. Comput. 38(157), 67-86 (1982)

3. Carpenter, M., Fisher, T., Nielsen, E., Frankel, S.: Entropy stable spectral collocation schemes for the Navier-Stokes equations: discontinuous interfaces. SIAM J. Sci. Comput. 36(5), B835-B867 (2014)

4. Gassner, G.: A skew-symmetric discontinuous Galerkin spectral element discretization and its relation to SBP-SAT finite difference methods. SIAM J. Sci. Comput. 35(3), A1233-A1253 (2013)

5. Gassner, G.J., Beck, A.D.: On the accuracy of high-order discretizations for underresolved turbulence simulations. Theoret. Comput. Fluid Dyn. 27(3-4), 221-237 (2013) 
6. Hesthaven, J.S., Kirby, R.M.: Filtering in Legendre spectral methods. Math. Comput. 77(263), 1425-1452 (2008)

7. Hesthaven, J.S., Warburton, T.: Nodal Discontinuous Galerkin Methods: Algorithms, Analysis, and Applications. Springer (2008)

8. Kennedy, C.A., Carpenter, M.H.: Comparison of Several Numerical Methods for Simulation of Compressible Shear Layers, Vol. 3484. NASA, Langley Research Center (1997)

9. Kopriva, D.A.: Implementing Spectral Methods for Partial Differential Equations. Springer, Scientific Computation (2009)

10. Kopriva, D.A., Gassner, G.J.: An energy stable discontinuous Galerkin spectral element discretization for variable coefficient advection problems. SIAM J. Sci. Comput. 36(4), A2076-A2099 (2014)

11. Lundquist, T., Nordström, J.: Stable and accurate filtering procedures. J. Sci. Comput. 82(1), 1-21 (2020)

12. Nordström, J.: A roadmap to well posed and stable problems in computational physics. J. Sci. Comput. 71(1), 365-385 (2016)

13. Nordström, J., Linders, V.: Well-posed and stable transmission problems. J. Comput. Phys. 364, 95-110 (2018)

14. Svärd, M., Nordström, J.: Review of summation-by-parts schemes for initial-boundary-value problems. J. Comput. Phys. 268, 17-38 (2014)

15. Tadmor, E., Zhong, W.: Energy-preserving and stable approximations for the two-dimensional shallow water equations. In: Mathematics and Computation, a Contemporary View, pp. 67-94. Springer (2008)

16. Toro, E.F.: Riemann Solvers and Numerical Methods for Fluid Dynamics: A Practical Introduction. Springer (2009)

17. Vandeven, H.: Family of spectral filters for discontinuous problems. J. Sci. Comput. 6(2), 159-192 (1991)

18. Williamson, J.H.: Low-storage Runge-Kutta schemes. J. Comput. Phys. 35, 48-56 (1980)

Publisher's Note Springer Nature remains neutral with regard to jurisdictional claims in published maps and institutional affiliations. 